\title{
The Origin of the Wigner Energy
}

\author{
W. Satuła ${ }^{1-3}$, D.J. Dean ${ }^{4}$, J. Gary ${ }^{2 *}$, S. Mizutori ${ }^{1,4}$, W. Nazarewicz ${ }^{2-4}$ \\ ${ }^{1}$ Joint Institute for Heavy Ion Research, Oak Ridge National Laboratory, P.O. Box 2008, \\ Oak Ridge, TN 37831, U.S.A. \\ ${ }^{2}$ Department of Physics, University of Tennessee, Knoxville, TN 37996, U.S.A. \\ ${ }^{3}$ Institute of Theoretical Physics, Warsaw University, ul. Hoża 69, PL-00681, Warsaw, Poland \\ ${ }^{4}$ Physics Division, Oak Ridge National Laboratory, P.O. Box 2008, Oak Ridge, TN 37831, U.S.A.
}

\begin{abstract}
Surfaces of experimental masses of even-even and odd-odd nuclei exhibit a sharp slope discontinuity at $N=Z$. This cusp (Wigner energy), reflecting an additional binding in nuclei with neutrons and protons occupying the same shell model orbitals, is usually attributed to neutron-proton pairing correlations. A method is developed to extract the Wigner term from experimental data. Both empirical arguments and shell-model calculations suggest that the Wigner term can be traced back to the isospin $T=0$ part of nuclear interaction. Our calculations reveal the rather complex mechanism responsible for the nuclear binding around the $N=Z$ line. In particular, we find that the Wigner term cannot be solely explained in terms of correlations between the neutron-proton $J=1, T=0$ (deuteron-like) pairs.
\end{abstract}

PACS numbers: 21.10.Dr, 21.10.Hw, 21.60.Cs

Typeset using REVTEX

*Science Alliance summer student from University of Missouri - Rolla. 
One of the most interesting avenues in nuclear structure research is the study of nuclear properties near the particle drip lines. Theoretically, due to their unusual shell structure and weak binding, nuclei with highest and lowest $N / Z$ ratios represent a unique challenge for the many-body problem.

This Letter concerns the proton-rich border of nuclear binding $(N / Z \approx 1)$. The proton drip line is relatively well known experimentally, and the discovery of ${ }^{100} \mathrm{Sn}$ indicates the potential that radioactive nuclear beams will access all bound $N=Z$ nuclei and their bound and unbound neighbors. This opens up the possibility of nuclear structure studies of mediummass and heavy systems with $N \approx Z$.

There are many aspects of nuclear structure which make physics near the $N=Z$ line very interesting: proton and diproton emission, isospin mixing, superallowed beta decay, and nuclear constraints for the astrophysical $r p$-process - all these are timely experimental and theoretical themes. A unique aspect of nuclei with $N=Z$ is that neutrons and protons occupy the same shell-model orbitals. Consequently, the large spatial overlaps between neutron and proton single-particle wave functions are expected to enhance neutron-proton $(n p)$ correlations, especially the $n p$ pairing.

At present, it is not clear what the specific experimental fingerprints of the $n p$ pairing are, whether the $n p$ correlations are strong enough to form a static condensate, and what their main building blocks are. Most of our knowledge about nuclear pairing comes from nuclei with a sizable neutron excess where the isospin $T=1$ neutron-neutron $(n n)$ and protonproton $(p p)$ pairing dominate. Now, for the first time, there is an experimental opportunity to explore nuclear systems in the vicinity of the $N=Z$ line which have many valence $n p$ pairs; that is, to probe the interplay between the like-particle and neutron-proton ( $T=0,1$, $\left.T_{z}=0\right)$ pairing channels.

This novel situation calls for the generalization of established theoretical models of nuclear pairing. In spite of several early attempts to extend the independent quasi-particle formalism to incorporate the effect of $n p$ correlations in light nuclei [1] [4] (see Ref. [5] for an early review), no symmetry-unrestricted calculations for $n p$ pairing, based on the quasiparticle theory, have been carried out.

So far, the strongest evidence for $n p$ pairing comes from the masses of $N=Z$ nuclei. An additional binding (the so-called Wigner energy) found in these nuclei manifests itself as a spike in the isobaric mass parabola as a function of $T_{z}=\frac{1}{2}(N-Z)$ (see the review [6] and Refs. quoted therein). Recently, it has been demonstrated that the $T=0 \mathrm{np}$-pair correlations, treated within the framework of the generalized quasi-particle theory, may produce such a cusp at $N=Z$ []]. Gross estimates of the magnitude of the Wigner energy come from a largescale fit to experimental binding energies with the macroscopic-microscopic approach [8.9] and from the analysis of experimental masses [10]. In this study, we present a technique to extract the Wigner energy directly from the experimental data, and give empirical arguments that this energy originates primarily from the $T=0$ part of the effective interaction. To obtain deeper insight into the structure of the Wigner term, we apply the nuclear shell model to nuclei from the $s d$ and $f p$ shells.

In the semi-empirical mass formulae (see e.g. Ref. [9]), an additional binding due to the $n p$-pair correlations is usually parameterized as

$$
B_{n p}^{\text {pair }}=-\epsilon_{n p}(A) \pi_{n p}+E_{W},
$$


where $\pi_{n p}=\frac{1}{4}\left(1-\pi_{n}\right)\left(1-\pi_{p}\right)$ and $\pi_{n}=(-1)^{N}$ and $\pi_{p}=(-1)^{Z}$ being the nucleonnumber parities. The first contribution to the $n p$ pairing energy in Eq. (1), $\epsilon_{n p}$, represents an additional binding due to the residual interaction between the two odd nucleons in an odd-odd nucleus.

The second contribution to $B_{n p}^{\text {pair }}, E_{W}$, dubbed the Wigner energy, is believed to represent the energy of collective $n p$-pairing correlations. It can be decomposed into two parts:

$$
E_{W}=W(A)|N-Z|+d(A) \pi_{n p} \delta_{N Z},
$$

The $|N-Z|$-dependence in Eq. (2) was first introduced by Wigner [1] in his analysis of the $\mathrm{SU}(4)$ spin-isospin symmetry of nuclear forces. In the supermultiplet approximation, there appears a term in the nuclear mass formula which is proportional to $T_{\mathrm{gs}}\left(T_{\mathrm{gs}}+4\right)$, where $T_{\mathrm{gs}}$ denotes the isospin of the ground state. Empirically, $T_{\mathrm{gs}}=\left|T_{z}\right|$ for most nuclei except for heavy odd-odd $N=Z$ systems [12,13]. Although the experimental data indicate that the $\mathrm{SU}(4)$ symmetry is severely broken, and the masses behave according to the $T_{\mathrm{gs}}\left(T_{\mathrm{gs}}+1\right)$ dependence [12.10], the expression of Eq. (2) for the Wigner energy is still very useful. In particular, it accounts for a non-analytic behavior of nuclear masses when an isobaric chain crosses the $N=Z$ line. An additional contribution to the Wigner term, the $d$-term in Eq. (2), represents a correction for $N=Z$ odd-odd nuclei. Theoretical justification of Eq. (2) has been given in terms of basic properties of effective shell-model interactions [6,14], and also by using simple arguments based on the number of valence $n p$-pairs [10,15]. The estimates based on the number of $n p$ pairs in identical spatial orbits suggest that the ratio $d / W$ is constant and equal to one [15]. A different estimate has been given in Ref. [10]: $d / W=0.56 \pm 0.27$.

In order to extract empirical information on the magnitude of pairing correlations, one usually applies mass relations (indicators, filters) defined as specific combinations of nuclear binding energies of neighboring nuclei [10]. The use of these filters is based on the following assumptions: $(i)$ the nuclear binding energy may be decomposed into a part $\tilde{B}$ which varies smoothly with $N$ and $Z$ (at least away from the closed shells) and a fluctuating (and possibly non-analytic) term; and (ii) the pairing energy may be parameterized as a smooth function of $N$ and $Z$ to reproduce global (large-scale) behavior. The important feature of mass indicators, based on finite-differences of the nuclear binding energy with respect to $N$ and $Z$, is that they remove the smooth energy background $\tilde{B}$ up to third [19,16] (or higher [17]) orders, and isolate specific parts of the pairing energy. However, the interpretation of results depends on the particular indicator used and the assumed parameterization of pairing energy.

The magnitude of the Wigner term is non-negligible only in the closest vicinity of the $N=Z$ line. However, since pairing indicators are given by finite differences, perturbations at $N=Z$ might disturb the physical interpretation of extracted quantities. For example, the well-known four-point odd-even mass difference formulae for the $n n$ and $p p$ pairing gaps are quite strongly perturbed near the $N=Z$ line [18]. Consequently, to isolate interesting effects, other indicators, involving more nuclei, must be used.

An indicator particularly useful for investigating the $n p$ correlation energy is the doubledifference formula of Ref. [19]:

$$
\delta V_{n p}(N, Z)=\frac{1}{4}\{B(N, Z)-B(N-2, Z)-B(N, Z-2)+B(N-2, Z-2)\}
$$




$$
\approx \frac{\partial^{2} B}{\partial N \partial Z}
$$

This indicator belongs to a different class than those analyzed in Ref. [10. First, it involves binding energies of nuclei having the same nucleon-number parity and, therefore, it is sensitive to the Wigner energy [20]. Second, indicator (3) does not remove the secondorder contribution to the smooth energy proportional to $N Z$. As a consequence, for nuclei with $T_{z}>1, \delta V_{n p}$ mainly probes the symmetry energy term, $\sim(N-Z)^{2} / A$, and for nuclei with $T_{z} \leq 1$ it contains both Wigner energy and symmetry energy contributions. The nuclear symmetry energy is well described by a self-consistent mean-field theory based on the realistic effective particle-hole interaction. Indeed, the application of indicator (3) to the theoretical self-consistent mass tables using either a spherical Hartree-Fock-Bogoliubov model (HFB) 21] or a deformed extended Thomas-Fermi plus Strutinsky-integral model (ETFSI) 22 gives results very consistent with the experimental values of $\delta V_{n p}$ for nuclei with $T_{z}>1$ [18]. This simple test indicates that the symmetry energy is rather insensitive to shell effects. Its strength smoothly varies with $A$ and is practically $T_{z}$-independent. Since, in this study, we are mainly interested in the Wigner energy, another indicator has to be employed that allows for filtering out the symmetry energy contribution. This can be achieved by considering combinations of $\delta V_{n p}$ values, as discussed in the following.

In this work, the Wigner energy coefficient $W$ in an even-even nucleus $Z=N=\frac{A}{2}$ has been extracted by means of the indicator:

$$
W(A)=\delta V_{n p}\left(\frac{A}{2}, \frac{A}{2}\right)-\frac{1}{2}\left[\delta V_{n p}\left(\frac{A}{2}, \frac{A}{2}-2\right)+\delta V_{n p}\left(\frac{A}{2}+2, \frac{A}{2}\right)\right] .
$$

The $d$-term in an odd-odd nucleus, $Z=N=\frac{A}{2}$ [Eq. (2) $]$ can be extracted using another indicator:

$$
d(A)=2\left[\delta V_{n p}\left(\frac{A}{2}, \frac{A}{2}-2\right)+\delta V_{n p}\left(\frac{A}{2}+2, \frac{A}{2}\right)\right]-4 \delta V_{n p}\left(\frac{A}{2}+1, \frac{A}{2}-1\right) .
$$

Although the recipe for these third-order mass difference indicators is not unique, the results appear to be very weakly dependent on the particular prescription used [18.

The experimental values of both contributions to the Wigner energy are shown in Fig. 1. The values of $W$ decrease rather smoothly with $A$, showing characteristic oscillations that are related to the shell effects. Indeed, the filling of $1 d_{5 / 2} 2 s_{1 / 2}$ shells results in a decrease of $W$ with the local minimum near $A \sim 34$. Subsequent filling of the $1 d_{3 / 2}$ shell tends to increase $W$. The next minimum in $W$ appears at the middle of the $1 f_{7 / 2}$ shell. Table $\mathbb{\square}$ shows the results of a least-squares fit to $W$ assuming $W(A)=a_{W} / A^{\alpha}$. The actual minimum corresponds to $\alpha \approx 0.95$; it is very close to the standard value of $\alpha=1$. The extracted strength $a_{W} \approx 47 \mathrm{MeV}$ is considerably larger than the one used in the semi-empirical mass formula of Ref. [9] $\left(a_{W}=30 \mathrm{MeV}\right)$, but is consistent with the value of Ref. [10] $\left(a_{W}=43 \pm 5 \mathrm{MeV}\right)$.

As seen in Fig. 1, for the $s d$ nuclei, the behavior of the $d$-term follows rather closely that of $W$, but this nice agreement is lost for the heavier systems. However, it is known experimentally (cf. discussion in Ref. [6] and references quoted therein) that as a rule the nuclear ground states have isospin $T_{\mathrm{gs}}=|N-Z| / 2$, except for heavy $N=Z$ odd-odd nuclei. Indeed, ${ }^{34} \mathrm{Cl}$ and nuclei heavier than ${ }^{40} \mathrm{Ca}$ (except for ${ }^{58} \mathrm{Cu}$ ) have ground states of isospin $T=1$. Replacing, in our analysis, the ground-state binding energies of these odd-odd $N=Z$, 
$T=1$ nuclei by the binding energies of their lowest $T=0$ states leads to modified $d$-values marked by triangles in Fig. 1. The resulting values follow very closely the values of $W$; i.e. $d_{T=0}(A) / W(A) \approx 1$, in a nice agreement with the simple arguments of Ref. [15]. This observation provides an important experimental argument that both terms constituting $E_{W}$ have the same microscopic origin. In particular, assuming that the structure of the lowest $T=0$ states in odd-odd $N=Z$ nuclei is strongly influenced by the effective interaction between $T=0$ pairs, the close similarity between $d_{T=0}$ and $W(A)$ indicates that the $T=0$ component of the nuclear interaction is responsible for the Wigner term.

The role of the $T=0$ part of the interaction on the presence of binding-energy irregularities in $\delta V_{n p}$ near the $N=Z$ has been recognized in Ref. [20]. They nicely illuminated this point by demonstrating that in the shell-model calculations for $s d$ nuclei using the surface delta interaction (SDI), it is indeed the $T=0$ part of SDI that give rise to the cusp in $\delta V_{n p}$.

In order to investigate the role played by the $T=0$ component for the Wigner energy, and to study the relative importance of various $n p$ pairs, we performed shell-model calculations in both the $1 f 2 p$ and $1 d 2 s$ shell-model spaces using the shell-model code ANTOINE [24]. In the $f p$-shell we use the KB3 effective interaction of Ref. [25], while for the $s d$-shell nuclei we use the USD effective interaction of Ref. [26]. The results of the calculations are displayed in Figs. 2 and 3.

The insert in Fig. 2 shows the calculated binding energies (solid circles) for the $A=48$ isobaric chain of even-even $f p$ nuclei relative to the $N=Z$ nucleus ${ }^{48} \mathrm{Cr}$. The Wigner term gives rise to an additional binding for the $N=Z$ nuclei. In Fig. 2, this is represented as a deviation, $\varepsilon_{W}$, from a smooth parabola fitted to the $N \neq Z$ isobars. Note, however, that the quantity, $\varepsilon_{W}$, is not a realistic estimate of the Wigner energy $E_{W}$, but a rather schematic measure of the cusp円.

To visualize the influence of the $T=0$ part of the effective nuclear interaction on the Wigner term, we have performed a set of shell-model calculations while switching off sequentially the $J=1,2, \ldots, J_{\max }, T=0$ two-body matrix elements $\left\langle j_{1} j_{2} J T|\hat{H}| j_{1}^{\prime} j_{2}^{\prime} J T\right\rangle$ of the shell-model Hamiltonian $\hat{H}$ for different values of $J_{\max }$. Figure 2 shows a ratio $\varepsilon_{W} / \varepsilon_{W}^{\text {total }}$, where $\varepsilon_{W}^{\text {total }}$ denotes the result of full shell-model calculations versus $J_{\max }$. The calculations were performed for two representative examples, namely, the $f p$-shell nucleus ${ }^{48} \mathrm{Cr}$ and the $s d$-shell nucleus ${ }^{24} \mathrm{Mg}$. The largest contribution to the Wigner energy comes from the part of the $T=0$ interaction between deuteron-like $(J=1)$ and 'stretched' $[J=5(s d)$ and 7 $(p f)]$ pairs. The importance of these matrix elements is well known; it is precisely for $J=1$ and stretched pair-states that experimentally determined effective $n p T=0$ interactions are strongest [27 29]. Note also that the deuteron-like correlations contribute more strongly to $\varepsilon_{W}$ in $s d$-nuclei than in $f p$-nuclei, and that matrix elements corresponding to intermediate values of $J$ give non-negligible contributions. This reveals the complex structure of the Wigner energy, and suggests that models which ignore high- $J$ components of the $n p$ interaction (e.g., by considering only $J=0, T=1$ and $J=1, T=0 n p$ pairs [30]) are not too

\footnotetext{
${ }^{1}$ In reality, there is some contribution from the collective $n p$-pairing effects also in nuclei with $|N-Z| \geq 4$, and, due to the isospin invariance of the interaction, the isotopic chains of even-even nuclei considered in Fig. 2 contain only three independent masses. Hence, the simple parabolic fit does not represent the realistic symmetry-energy dependence.
} 
useful for discussing the actual $n p$ pair correlations.

Since in the self-consistent models based on the realistic effective interactions the explicit $n p$ coupling is ignored, they cannot reproduce the Wigner term (see, however, Ref. [7]; for the recent extension of the HFB method to $n p$ pairing, see Ref. [31]). The successful ETFSI mass formula, based on Skyrme-like particle-hole interaction and monopole-pairing interaction between like nucleons, accounts well for most effects attributed to the particlehole channel, e.g., shell effects and deformations. However, all those are filtered out by indicator (4). This is illustrated in the insert to Fig. 3 which shows that $W \approx 0$ in ETFSI.

The $0 \hbar \omega$ shell model, on the other hand, reproduces well experimental values of $W$ both in the $s d$ - as well as in the $f p$-shell (circles in Fig. 3). The results of calculations with the $(J=1, T=0)$ two-body matrix elements removed, marked by triangles in Fig. 33, demonstrate that the impact of the deuteron-like correlations on the strength of the Wigner term is stronger in $s d$-nuclei than in $f p$-nuclei (cf. also Fig. 2). We also note the strong variations in the region of $A \sim 32$ where $W$ becomes locally negative. These variations are associated with the subsequent filling of the upper part of the $1 d_{5 / 2}$, the $2 s_{1 / 2}$, and of the bottom part of the $1 d_{3 / 2}$ shells. In the extreme shell-model picture, both $1 d_{5 / 2}$ and $2 s_{1 / 2}$ shells are occupied for $Z=N=16$, and the $d_{3 / 2}$ shell is well separated. Consequently, the important contribution to $W$ comes from $T=0$ matrix elements with $j_{n} \neq j_{p}$ ).

Removing all $T=0$ matrix elements with $J=1,3,5,7$ ( $J_{\max }^{\text {odd }}=7$ variant), i.e. those which are predominantly due to the coupling between neutrons and protons in identical orbits, washes out $W$ almost completely. An exception is the upper part of the $s d$-shell $\left(d_{3 / 2}\right.$ subshell) where the value of $W$ is reduced by about $50-60 \%$. Intuitively, below ${ }^{40} \mathrm{Ca}$ one can expect a quenching of phase-space available for scattering the $n p$-pairs in identical orbits. Simultaneously, one would expect a slight increase of the role of $j_{n} \neq j_{p} n p$-pairs. Indeed, removing the remaining $T=0$ matrix elements with even values of $J$ ( $J_{\max }=7$ variant) has the largest impact on the heaviest $s d$-shell nuclei.

We briefly comment on the first term, $\varepsilon_{n p}(A)$, in Eq. (11). The magnitude of the residual $n p$ correlation between the last nucleons in an odd-odd system may be extracted from experimental masses using the pairing indicators $\Delta_{n p(p n)}$ proposed in Ref. [10]. In the semiempirical mass formulae, the most common parameterization of $\varepsilon_{n p}(A)$ assumes a volumelike dependence of $\varepsilon_{n p}(A) \approx \varepsilon / A$ with $\varepsilon \approx 20-40 \mathrm{MeV}$ [9,10]. Our analysis performed for $A \geq 20$ shows that the $\varepsilon_{n p}(A)$ is almost mass-independent for nuclei around the $N=Z$ line (with $|N-Z| \leq 3$ ), and is of the order of $\varepsilon_{n p}(A) \approx 500 \mathrm{keV}$. Therefore, particularly for light nuclei, the magnitude of $\varepsilon_{n p}$ is 2-3 times smaller than suggested in Refs. 99,10]. For heavier nuclei with larger neutron excess, $\varepsilon_{n p}$ is systematically weaker and approaches the value of $\sim 300 \mathrm{keV}$.

In summary, we performed a systematic study of $n p$-pair correlations in $N \sim Z$ nuclei. Our main emphasis has been the analysis of the Wigner term in terms of $n p$ pairs. The experimental data are consistent with a two-term structure given by Eq. (2)). We developed a reliable method which allows for the extraction of both $W$ and $d$ components of the Wigner energy from experimental data. Experimental values are consistent with the simple relation $d_{T=0} / W \approx 1$. This is an empirical argument that the Wigner term emerges from the $T=0$ part of the $n p$ pair interaction. The presence of an extra correction to the binding energy of odd-odd nuclei (the $d$-term) may have important consequences for the independent quasiparticle theory of $n p$ pairing where even-even and odd-odd nuclei are treated on the same 
footing.

To investigate the microscopic structure of the Wigner term, we performed shell-model calculations for even-even nuclei while attenuating, in various ways, the $T=0$ part of the effective shell-model interaction. This study confirms that the bulk part of Wigner energy comes from the $T=0 \mathrm{np}$ pairs. We also found that the contribution from deuteron-like $(J=1$, $T=0)$ pairs is by no means dominant; it is similar to, and near the middle of the $f_{7 / 2}$-shell even weaker than, the contribution from maximally aligned pairs. The contributions from the $T=0$ pairs with intermediate spins are also non-negligible, especially in the upper part of the $s d$-shell. Finally, we found that the residual interaction between the valence proton and the valence neutron in an odd-odd nucleus exhibits a very weak mass dependence.

\section{ACKNOWLEDGMENTS}

The authors would like to thank Jacek Dobaczewski for useful comments. This research was supported in part by the U.S. Department of Energy under Contract Nos. DE-FG0296ER40963 (University of Tennessee), DE-FG05-87ER40361 (Joint Institute for Heavy Ion Research), DE-AC05-96OR22464 with Lockheed Martin Energy Research Corp. (Oak Ridge National Laboratory), and by the Polish Committee for Scientific Research under Contract No. 2 P03B 034 08. DJD acknowledges a Wigner fellowship from ORNL. 


\section{REFERENCES}

[1] A. Goswami, Nucl. Phys. 60 (1964) 228.

[2] A. Goswami and L.S. Kisslinger, Phys. Rev. 140 (1965) B26.

[3] H.T. Chen and A. Goswami, Phys. Lett. B24 (1967) 257.

[4] H. Wolter, A. Faessler and P. Sauer, Nucl. Phys. A167 (1971) 108.

[5] A.L. Goodman, Adv. Nucl. Phys. 11 (1979) 263.

[6] N. Zeldes, in Handbook of Nuclear Properties, Ed. by D. Poenaru and W. Greiner, Clarendon Press, Oxford, 1996, p. 13.

[7] W. Satuła and R. Wyss, Phys. Lett. B, in print.

[8] W.D. Myers and W.J. Swiatecki, Nucl. Phys. A81 (1966) 1.

[9] H.J. Krappe, J.R. Nix and A.J. Sierk, Phys. Rev. C20 (1979) 992.

[10] A.S. Jensen, P.G. Hansen and B. Jonson, Nucl. Phys. A431 (1984) 393.

[11] E.P. Wigner, Phys. Rev. 51 (1937) 106.

[12] J. Jänecke, Nucl. Phys. 73 (1965) 97.

[13] N. Zeldes and S. Liran, Phys. Lett. 62B (1976) 12.

[14] I. Talmi, Rev. Mod. Phys. 34 (1962) 704.

[15] W.D. Myers, Droplet model of atomic nuclei, Plenum, NY, 1977.

[16] N. Zeldes, M. Gronau, and A. Lev, Nucl. Phys. 63 (1965) 1.

[17] P. Möller and R. Nix, Nucl. Phys. A536 (1992) 20.

[18] W. Satuła, D. Dean, J. Dobaczewski, J. Gary, J.D. Garrett, S. Mizutori, and W. Nazarewicz, in preparation.

[19] J.-Y. Zhang, R.F. Casten and D.S. Brenner, Phys. Lett. 227B (1989) 1.

[20] D.S. Brenner, C. Wesselborg, R.F. Casten, D.D. Warner and J.-Y. Zhang, Phys. Lett. 243B (1990) 1.

[21] J. Dobaczewski, W. Nazarewicz, and T.R. Werner, Z. Phys. A354 (1996) 27.

[22] Y. Aboussir, J.M. Pearson, A.K. Dutta and F. Tondeur, Atomic Data and Nuclear Data Tables 61 (1995) 127.

[23] G. Audi and A.H. Wapstra, Nucl. Phys. A595 (1995) 409.

[24] E. Caurier, code ANTOINE, Strasbourg, (1989).

[25] A. Poves and A.P. Zucker, Phys. Rep. 70 (1981) 235.

[26] B.H. Wildenthal, Prog. in Part. and Nucl. Phys. 11 (1984) 5.

[27] N. Anantaraman and J.P. Schiffer, Phys. Lett. 37B (1971) 229.

[28] J.P. Schiffer, Ann. Phys. 66 (1971) 78.

[29] A. Molinari, M.B. Johnson, A.A. Bethe, and W.M. Alberico, Nucl. Phys. A239 (1975) 45.

[30] J.A. Evans, G.G. Dussel, E.E. Maqueda, and R.P.J. Perazzo, Nucl. Phys. A367 (1981) 77.

[31] E. Perlinska, S.G. Rohozinski, J. Dobaczewski, and W. Nazarewicz, Proc. Int. Conf. Extremes of Nuclear Structure, Hirschegg'96, ed. by H. Feldmeier, J. Knoll, and W. Noerenberg (FIZ Karlsruhe, 1996) p. 228.

[32] E.P. Wigner, Group Theory, Academic Press, New York, 1959.

[33] N. Zeldes, A. Grill and A. Simievic, Mat. Fys. Skr. Dan. Vid. Selsk. 3 (1967) 1. 


\section{TABLES}

TABLE I. Results of the least-squares fits for the Wigner energy strength $W(A)=a_{W} / A^{\alpha}$ for $\alpha=1 / 2,2 / 3$, and 1 . The actual minimum of the mean standard deviation $\sigma$ corresponds to $\alpha=0.92$ $(\sigma \approx 0.196)$. All data points with $Z \geq 10$ shown in Fig. 11 were considered in the fit.

\begin{tabular}{rrr}
\hline \hline$\alpha$ & $a_{W}$ & $\sigma_{W}$ \\
& $\mathrm{MeV}$ & $\mathrm{MeV}$ \\
\hline $1 / 2$ & 8 & 0.239 \\
$2 / 3$ & 14 & 0.213 \\
1 & 47 & 0.196 \\
\hline \hline
\end{tabular}




\section{FIGURES}

FIG. 1. Experimental values of $W$ (filled circles) and $d$ [Eq. (5), open circles] in $N=Z$ nuclei extracted from measured binding energies [23]. For even-even nuclei, the values of $W$ were obtained from Eq. (四), while the values for odd-odd nuclei were obtained by means of a similar indicator, involving binding energies of $N=Z$ and $N=Z+2$ even-even cores. The triangles mark the values of $d$ calculated using experimental binding energies of the lowest $T=0$ states in odd-odd nuclei. The solid line represents the average value $W_{\mathrm{av}}=47 / A$ (see text).

FIG. 2. Calculated displacement $\varepsilon_{W}$ of the binding energy of ${ }^{24} \mathrm{Mg}$ and ${ }^{48} \mathrm{Cr}$ from the average parabolic $(N-Z)^{2}$ behavior along an isobaric chain. Shell-model calculations were performed in the $0 \hbar \omega$ configuration space. The results of calculations for the binding energies of even-even nuclei along the $A=48$ chain (normalized to ${ }^{48} \mathrm{Cr}$ ) are shown in the insert. The values of $\varepsilon_{W}$ were obtained using the shell-model Hamiltonian with the $J=1,2, . ., J_{\max }, T=0$ matrix elements removed. For instance, the result for $J_{\max }=3$ corresponds to the variant of calculations in which all the two-body matrix elements between states $\left|j_{1} j_{2} J T=0\right\rangle$ with $J=1,2,3$, were put to zero. The results are normalized to the full shell-model value $\varepsilon_{W}^{\text {total }}\left(J_{\max }=0\right)$. The Coulomb contribution to the binding energy has been disregarded.

FIG. 3. The strength of the Wigner term, $W$, extracted using binding energies calculated with the $0 \hbar \omega$ shell model. Full shell-model calculations (filled circles) agree very well with experimental data (open circles). The results of shell-model calculations with the $(J=1, T=0)$ two-body matrix elements removed $\left(J_{\max }=1\right.$ variant, triangles), with all $T=0$ matrix elements removed $\left(J_{\max }=7\right.$ variant, diamonds), and with $(J=1,3,5,7, T=0)$ two-body matrix elements removed ( $J_{\max }^{\text {odd }}=7$ variant, squares) are also shown. In the $f p$-shell, only two points have been calculated due to practical limitations. Also a bridge between $s d$ and $f p$ shells cannot be covered as it requires interactions that include cross-shell matrix elements which are currently not established. The insert shows the values of $W$ extracted from the ETFSI mass formula [22]. They are practically zero for all nuclei considered. 


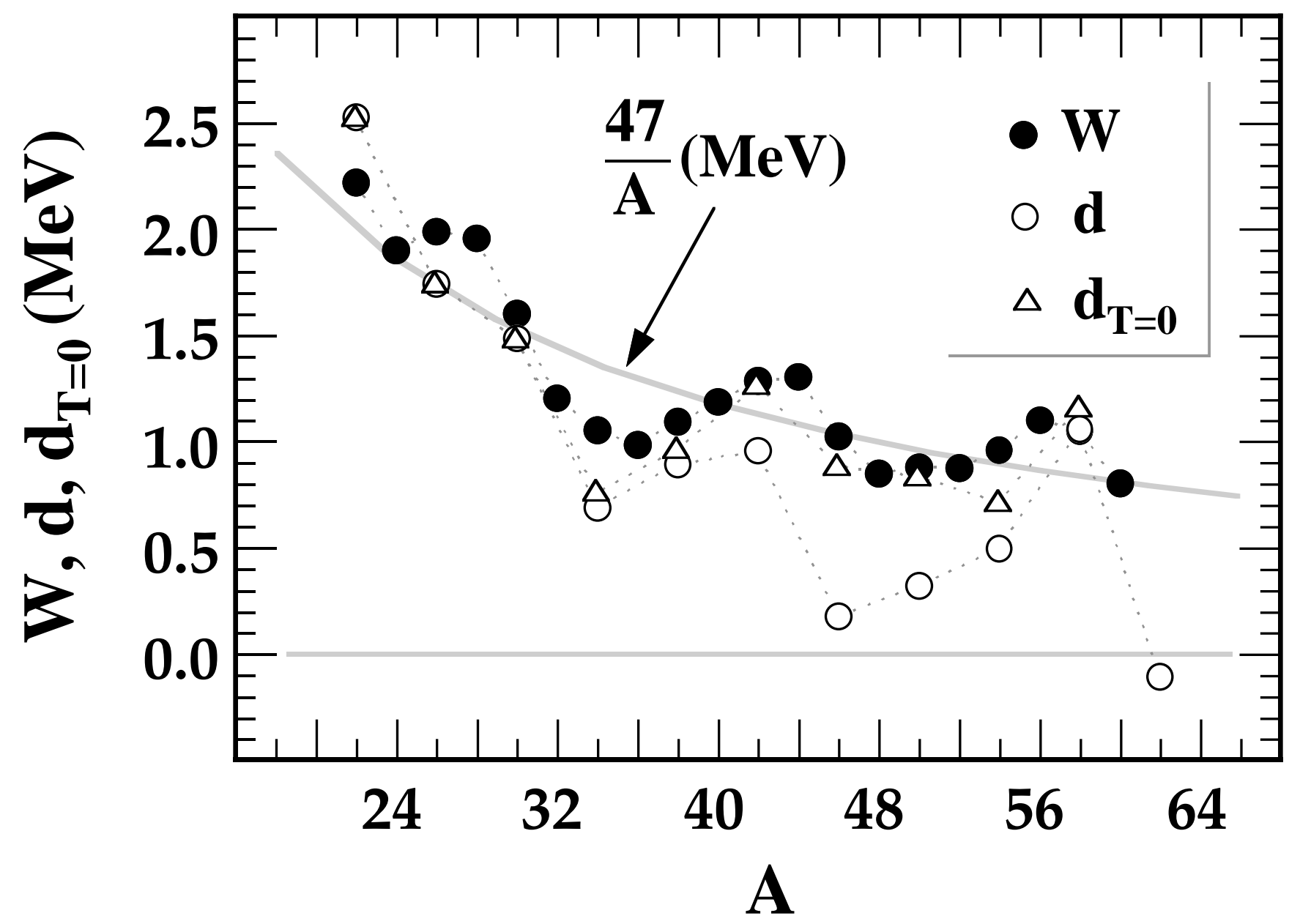

W. Satula et al., Fig. 1 


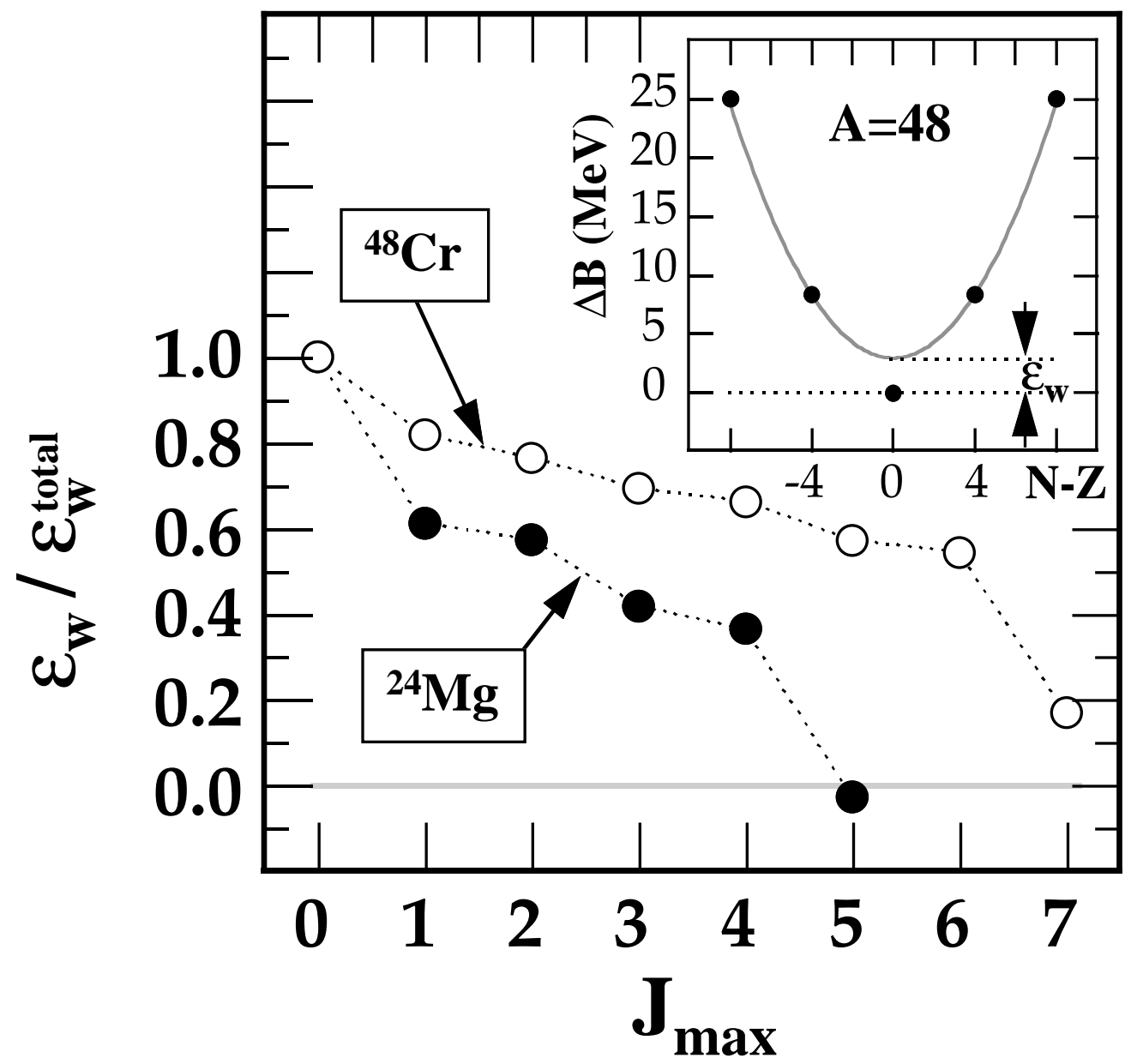

W. Satula et al., Fig. 2 


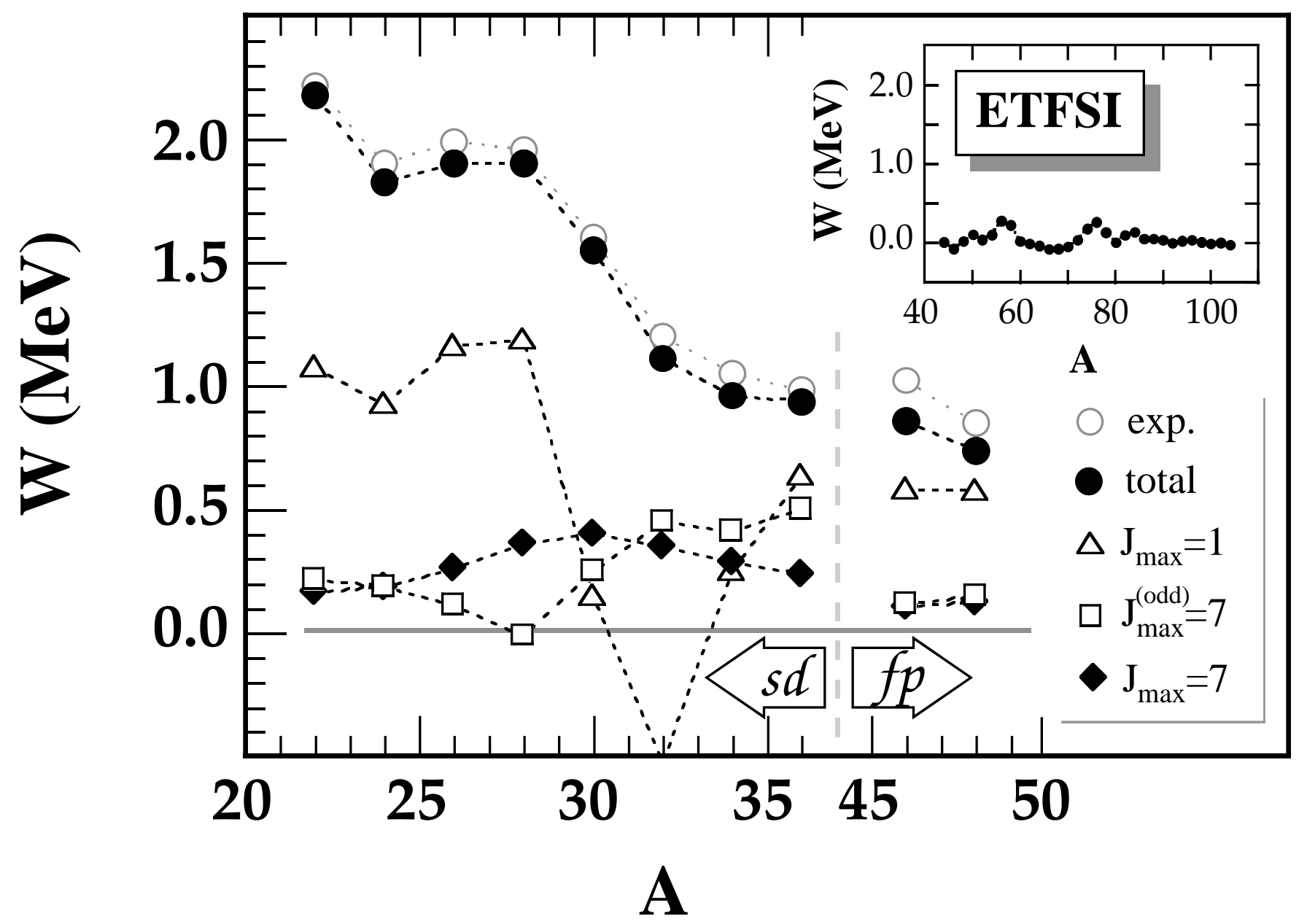

W. Satula et al., Fig. 3 